ALEA, Lat. Am. J. Probab. Math. Stat. 15, 1241-1255 (2018)

DOI: 10.30757/ALEA.v15-46

\title{
Characterization of non-commutative free Gaussian variables
}

\section{Arup Bose, Apratim Dey and Wiktor Ejsmont}

\author{
Indian Statistical Institute \\ 203 B.T. Road, \\ Kolkata 700108 \\ India \\ E-mail address: bosearu@gmail.com \\ URL: https://www.isical.ac.in/ abose/ \\ Indian Statistical Institute \\ 203 B.T. Road, \\ Kolkata 700108 \\ India \\ E-mail address: apratimdey.isical@gmail.com
}

Mathematical Institute University of Wroclaw

pl. Grunwaldzki 2/4,

50-384 Wroclaw,

Poland

E-mail address: wiktor.ejsmont@gmail.com

URL: https://sites.google.com/site/wiktorejsmont/

\begin{abstract}
We provide a necessary and sufficient condition, based on zero correlation, for self-adjoint, freely independent, identically distributed random variables on a $*$-probability space to be free Gaussian. Along the way, we establish a free analogue of a well known application of Basu's theorem from statistics. We also show that all linear combinations of free Gaussian being free Gaussian does not necessarily imply joint free Gaussianity, and we identify additional conditions under which this implication is true.
\end{abstract}

Received by the editors November 19th, 2017; accepted September 24th, 2018.

2010 Mathematics Subject Classification. Primary 46L54, secondary 62E10.

Key words and phrases. Basu's theorem, free independence, free Gaussian, free cumulants, Möbius function, polynomial identity, operator-valued non-commutative probability space.

Arup Bose has been supported by the J. C. Bose Fellowship of the Govt. of India and Wiktor Ejsmont was supported by the Narodowe Centrum Nauki grant 2014/15/B/ST1/00064 and the Narodowe Centrum Nauki grant 2016/21/B/ST1/00628. 


\section{Introduction}

Free probability was introduced by Voiculescu (1986) 30 years ago in order to solve some problems in von Neumann algebras of free groups and has since developed into a new field of mathematics with numerous connections to established fields such as classical probability, combinatorics and analysis, in particular to random matrices (see Voiculescu, 1991), non-crossing partitions (see Speicher, 1998), and operator algebras.

The study of free analogues of classical theorems in probability has witnessed increasing interest recently, see Arizmendi and Jaramillo (2014); Bożejko and Bryc (2006); Ejsmont et al. (2017); Ejsmont and Lehner (2017); Pardo et al. (2017); Hasebe (2016); Ejsmont (2014); Szpojankowski (2015, 2017); Szpojankowski and Wesołowski (2014). Many properties of free random variables are analogous to those of their classical counterparts, in particular when they are picked according to the Bercovici-Pata bijection. There are, however, exceptions, too. Our focus in this article lies on some results related to the characterization of Gaussian variables in classical probability and investigate whether analogous results are true for free Gaussian variables. We consider the following three well known results from classical probability.

1. Application of Basu's theorem. One of the most famous results in statistics, known as Basu's theorem (see Basu, 1955), says that a complete sufficient statistic and any ancillary statistic are stochastically independent. Perhaps its most important application is the following. For random variables $X_{i}, 1 \leq i \leq n$, let

$$
\bar{X}_{k}:=\frac{1}{k} \sum_{i=1}^{k} X_{i} \text { and } S_{k}:=\frac{1}{k} \sum_{i=1}^{k}\left(X_{i}-\bar{X}_{k}\right)^{2} \quad \text { for } \quad 2 \leq k \leq n .
$$

Theorem 1.1 (Application of Basu's theorem, see Basu, 1955). Suppose $X_{i}, 1 \leq$ $i \leq n$ are i.i.d. $N\left(\mu, \sigma^{2}\right)$. Then $\bar{X}_{n}$ is independent of $\left\{S_{2}, S_{3}, \ldots, S_{n}\right\}$.

2. Seneta and Szekely theorem (see Seneta and Szekely, 2006).

Theorem 1.2. Suppose $X_{i}, i=1, \ldots, n$ are independent and identically distributed random variables for some $n \geq 2$ with $E\left(X_{1}\right)=\mu, \operatorname{Var}\left(X_{1}\right)=\sigma^{2}$ and finite $(k+2)$ th moment for some integer $k>1$. Then

$$
\operatorname{Cov}\left(\left(\bar{X}_{n}-\mu\right)^{j}, S_{n}\right)=0, j=1,2, \ldots, k,
$$

implies that the first $k+2$ moments of $X_{1}$ coincide with those of an $N\left(\mu, \sigma^{2}\right)$ distributed random variable. In particular if all moments of $X_{1}$ are finite and (1.1) holds for all $k \geq 1$, then $X_{1}$ has the $N\left(\mu, \sigma^{2}\right)$ distribution.

Note that Theorem 1.2 provides a sort of converse to Theorem 1.1 under the additional assumption that all moments are finite.

3. Zero correlation and independence.

Theorem 1.3. Suppose that the random vector $[X, Y]$ has a bivariate normal distribution. If $\operatorname{Cov}(X, Y)$ is zero, then $X$ and $Y$ are independent.

In non-commutative (free) probability, the analogues of classical independence, Gaussian family of random variables, and Gaussian distribution, are respectively, 
free independence, free Gaussian family (semicircular family) of non-commutative random variables, and the semicircle distribution. A natural question is if analogues of the above results are true in the free world. The goal of this paper is to investigate these issues.

We show that the free analogues of Theorems 1.1 and 1.2 hold. Then we give an example to show that the natural free analogue of Theorem 1.3 is false. Nevertheless, an appropriate non-commutative (quantum) version of this result is true.

\section{Preliminaries}

A tracial non-commutative probability space is a pair $(\mathcal{A}, \tau)$ where $\mathcal{A}$ is a von Neumann algebra, and $\varphi: \mathcal{A} \rightarrow \mathbb{C}$ is a normal, faithful, tracial state, i.e. $\varphi$ is linear and continuous in the weak-* topology, and for all $X, Y \in \mathcal{A}, \varphi(X Y)=\varphi(Y X)$, $\varphi(\mathrm{I})=1, \varphi\left(X X^{*}\right) \geq 0$ and $\varphi\left(X X^{*}\right)=0$ implies $X=0$.

The self-adjoint elements in $\mathcal{A}$ are called non-commutative random variables. The distribution of any random variable $X$ (in the state $\varphi$ ) is the collection of its moments $\varphi\left(X^{n}\right), n \geq 1$, or equivalently, the unique probability measure $\mu_{X}$ on $\mathbb{R}$ (given by the spectral theorem) which satisfies $\varphi\left(X^{n}\right)=\int_{\mathbb{R}} \lambda^{n} d \mu_{X}(\lambda)$ for $n \in \mathbb{N}$.

A family of von Neumann sub-algebras $\left(\mathcal{A}_{i}\right)_{i \in I}$ of $\mathcal{A}$ is called free if $\varphi\left(X_{1} \cdots X_{n}\right)=$ 0 , whenever $\varphi\left(X_{j}\right)=0$ for all $j=1, \ldots, n$ and $X_{j} \in \mathcal{A}_{i(j)}$ for some indices $i(1) \neq i(2) \neq \cdots \neq i(n)$. Random variables $X_{1}, \ldots, X_{n}$ are said to be freely independent (in short free) if the sub-algebras they generate are free.

The joint distribution of any collection of random variables is the set of moments of all monomials formed from this collection. It is well known (see Mingo and Speicher, 2017, Ch.1, Prop. 13, or Nica and Speicher, 2006, Lemma 5.13) that the joint distribution of free random variables $\left\{X_{i}\right\}$ is uniquely determined by the distributions of the individual random variables $X_{i}$ and therefore the operation of free convolution is well defined. Let $\mu$ and $\nu$ be probability measures on $\mathbb{R}$, and let $X, Y$ be self-adjoint, free random variables with respective distributions $\mu$ and $\nu$. The distribution of $X+Y$ is called the free additive convolution of $\mu$ and $\nu$, and is denoted by $\mu \boxplus \nu$.

Let $\pi=\left\{V_{1}, \ldots, V_{p}\right\}$ be a partition of the linearly ordered set $1, \ldots, n$, i.e. the $V_{i} \neq \emptyset$ are ordered and disjoint sets whose union is $\{1, \ldots, n\}$. Then $\pi$ is called non-crossing if $a, c \in V_{i}$ and $b, d \in V_{j}$ with $a<b<c<d$ implies $i=j$. The set of all non-crossing partitions of the set $\{1, \ldots, n\}$ is denoted by $N C(n)$. The maximal element of $N C(n)$ under this order is the partition $\hat{1}_{n}$ consisting of only one block. The subset $N_{2}(n)$ of $N C(n)$ denotes the set of all non-crossing pair partitions, i.e. partitions where every block has size 2 . Let

$$
C_{n}=(n+1)^{-1}\left(\begin{array}{c}
2 n \\
n
\end{array}\right) \text { be the } n \text {-th Catalan number. }
$$

The following fact will be used repeatedly.

Fact 1. For every integer $n \geq 1, N C(n)$ and $N C_{2}(2 n)$ are in bijection and $|N C(n)|=C_{n}$. 
The free cumulants are multilinear maps $\kappa_{r}: \mathcal{A}^{r} \rightarrow \mathbb{C}$ defined implicitly by the relation (connecting them uniquely with mixed moments)

$$
\varphi\left(X_{1} X_{2} \cdots X_{n}\right)=\sum_{\pi \in N C(n)} \kappa_{\pi}\left(X_{1}, X_{2}, \ldots, X_{n}\right),
$$

where

$$
\kappa_{\pi}\left(X_{1}, X_{2}, \ldots, X_{n}\right):=\Pi_{B \in \pi} \kappa_{|B|}\left(X_{i}: i \in B\right) .
$$

Relation (2.1), upon inversion, means that for $\pi \in N C(n)$,

$$
\kappa_{n}\left(X_{1}, \ldots, X_{n}\right)=\sum_{\pi \in N C(n)} \operatorname{Möb}_{N C}\left(\pi, \hat{1}_{n}\right) \varphi_{\pi}\left(X_{1}, X_{2}, \ldots, X_{n}\right),
$$

where Möb ${ }_{N C}$ is the Möbius function on the lattice of non-crossing partitions and $\varphi_{\pi}$ denotes the multiplicative extension of the moments, as in (2.2). Sometimes we will write $\kappa_{r}(X)=\kappa_{r}(X, \ldots, X)$. Using these relations it is easy to prove the following fact. We shall use this repeatedly.

Lemma 2.1. For any variable $X$, all its odd moments up to order $k$ are zero if and only if all its odd free cumulants up to order $k$ are zero.

It follows immediately from above that

$$
\kappa_{1}(X)=\varphi(X) \text { and } \operatorname{Cov}(X, Y):=\kappa_{2}(X, Y)=\varphi(X Y)-\varphi(X) \varphi(Y) .
$$

The numbers $\kappa_{2}(X)=\varphi\left(X^{2}\right)-[\varphi(X)]^{2}$ and $\kappa_{2}(X, Y)$ are known as the variance of $X$ and covariance of $X, Y$, respectively. Free cumulants are called mixed, if they involve at least two different variables, else they are called pure.

Free cumulants provide the most important technical tool to investigate free random variables. Free independence of $X_{1}, \ldots, X_{n}$ is equivalent to saying that all their mixed free cumulants are zero. By this we mean that

$$
\kappa_{j}\left(X_{i_{1}}, X_{i_{2}}, \ldots, X_{i_{j}}\right)=0
$$

for $j \geq 2$, whenever at least two indices are different. A non-commutative random variable $X$ is said to be (standard) free Gaussian, or equivalently (standard) semicircle, if its moments are given by the formula

$$
\varphi\left(X^{k}\right)=\left\{\begin{array}{l}
C_{n}, \quad \text { if } k=2 n, \\
0, \quad \text { if } k \text { is odd } .
\end{array}\right.
$$

The free cumulants of this distribution satisfies $\kappa_{i}(X)=0$ for $i>2$. Recall that for a standard Gaussian variable, the classical cumulants of order greater than 2 vanish.

A set of self-adjoint variables $X_{1}, \ldots, X_{n}$ will be called a semicircular family if all mixed free cumulants of order three or higher are zero, and each $X_{i}$ is free Gaussian.

We define $[n]:=\{1, \ldots, n\}$. Following $[8$, Section 2.1, p.35], we define for a multi-index $\underline{i}=\left(i_{1}, i_{2}, \ldots, i_{m}\right) \in[n]^{m}$, ker $\underline{i}$ to be that partition $\pi \in P(m)$ (the set of all partitions of $[m])$ such that $i_{k}=i_{p}$ if and only if $k \stackrel{\pi}{\sim} p$ i.e. $k$ and $l$ are in the same block of $\pi$. We denote by $\underline{i} \circ \underline{j} \in[n]^{m+m^{\prime}}$ the concatenation of the multi-indices $\underline{i} \in[n]^{m}$ and $\underline{j} \in[n]^{m^{\prime}}$. 


\section{Main results}

\subsection{Free analogue of Theorem 1.1.}

Theorem 3.1. Fix an integer $n \geq 2$. Let $X_{1}, \ldots, X_{n}$ be a semicircular family where $\kappa_{2}\left(X_{i}, X_{i}\right)=\sigma^{2}$ for all $1 \in\{1, \ldots, n\}$ and $\kappa_{2}\left(X_{i}, X_{j}\right)=c$ for $1 \leq i \neq j \leq n$. Then $\bar{X}_{n}$ is free from $\left\{S_{2}, \ldots, S_{n}\right\}$.

Proof: The line of argument given below, would also suffice as a proof for the classical case. First note that

$$
\sum_{1 \leq i \neq j \leq r}\left(X_{i}-X_{j}\right)^{2}=2 r \sum_{i=1}^{r}\left(X_{i}-\overline{X_{r}}\right)^{2}=2 r^{2} S_{r}
$$

Hence, it suffices to demonstrate that $\bar{X}_{n}$ and the set of variables $\left\{X_{i}-X_{j}: i \neq\right.$ $j\}$ are free. Since $X_{1}, \ldots, X_{n}$ is a semicircular family, every collection of linear transformations, in particular $\left\{\bar{X}_{n}, X_{i}-X_{j}: i \neq j\right\}$, is also a semicircular family. Thus any mixed free cumulant of order 3 or higher is zero. It remains to be shown that any mixed free cumulant of order 2 of $\bar{X}_{n}$ and $X_{i}-X_{j}$ is zero. But this is easy to see, since by multilinearity of free cumulants and by traciality of $\varphi$

$$
\begin{aligned}
\kappa_{2}\left(\bar{X}_{n}, X_{i}-X_{j}\right) \\
\quad=\frac{1}{n}\left[\kappa_{2}\left(X_{i}, X_{i}\right)-\kappa_{2}\left(X_{j}, X_{j}\right)+\sum_{k \neq i} \kappa_{2}\left(X_{k}, X_{i}\right)-\sum_{k \neq j} \kappa_{2}\left(X_{k}, X_{j}\right)\right]=0 .
\end{aligned}
$$

Thus, $\bar{X}_{n}$ is free from $\left\{X_{i}-X_{j}, i \neq j\right\}$ and the theorem is proved.

3.2. Free analogue of Theorem 1.2. Note that the Seneta-Szekely result needs only a few moments. However, by default in our setup all moments are assumed to be finite. With this caveat, our next theorem is a converse to Theorem 3.1 and is a free analogue of Theorem 1.2.

Theorem 3.2. Let $X_{1}, \ldots, X_{n}$ be self-adjoint freely independent identically distributed random variables on $(\mathcal{A}, \varphi)$ with $\mu=\varphi\left(X_{1}\right), \operatorname{Var}\left(X_{1}\right)=\sigma^{2}$, such that

$$
\operatorname{Cov}\left((\bar{X}-\mu)^{r}, S_{n}\right)=0, \forall r \in \mathbb{N} .
$$

Then $\sigma^{-1}\left(X_{1}-\mu\right)$ has standard semicircular distribution.

Proof: Without loss of generality we assume that $\mu=0, \sigma=1$. We just need to prove that the moments of $X_{1}$ satisfy (2.4). The proof proceeds by induction on $k$. Note that $\varphi\left(X_{1}\right)=0, \varphi\left(X_{1}^{2}\right)=1$ and hence, (2.4) holds for $k=1,2$. In view of (3.1), (3.2) can be rewritten as

$$
\kappa_{2}\left(\left[\sum_{i=1}^{n} X_{i}\right]^{r}, \sum_{1 \leq i \neq j \leq r}\left(X_{i}-X_{j}\right)^{2}\right)=0, \forall r \in \mathbb{N} .
$$

Hence, using the identical distribution property of $X_{i}$ 's and the multilinearity of $\kappa_{2}$, we get

$$
\kappa_{2}\left(\left[\sum_{i=1}^{n} X_{i}\right]^{r},\left(X_{1}-X_{2}\right)^{2}\right)=0, \forall r \in \mathbb{N}
$$


This yields

$$
\begin{aligned}
& \varphi\left(\left[\sum_{i=1}^{n} X_{i}\right]^{r}\left(X_{1}^{2}-X_{1} X_{2}-X_{2} X_{1}+X_{2}^{2}\right)\right) \\
& =\varphi\left(\left[\sum_{i=1}^{n} X_{i}\right]^{r}\right) \varphi\left(X_{1}^{2}-X_{1} X_{2}-X_{2} X_{1}+X_{2}^{2}\right) .
\end{aligned}
$$

By hypothesis,

$$
\varphi\left(X_{1}^{2}\right)=\varphi\left(X_{2}^{2}\right)=1 \text { and } \varphi\left(X_{1} X_{2}\right)=\varphi\left(X_{2} X_{1}\right)=0 .
$$

Thus

and therefore,

$$
\varphi\left(X_{1}^{2}-X_{1} X_{2}-X_{2} X_{1}+X_{2}^{2}\right)=2,
$$

$$
\varphi\left(\left[\sum_{i=1}^{n} X_{i}\right]^{r}\left(X_{1}^{2}-X_{1} X_{2}-X_{2} X_{1}+X_{2}^{2}\right)\right)=2 \varphi\left(\left[\sum_{i=1}^{n} X_{i}\right]^{r}\right)
$$

for all positive integers $r$.

We shall use the above relation to first show that the odd moments of $X_{1}$ are zero, and then we will evaluate the even moments. Suppose that $\varphi\left(X_{1}^{2 r-1}\right)=0$ for $1 \leq r \leq m$. We intend to show that $\varphi\left(X_{1}^{2 m+1}\right)=0$. It follows from freeness and Lemma 2.1, that

$$
\begin{aligned}
\varphi\left(\left[\sum_{i=1}^{n} X_{i}\right]^{2 m-1}\right) & =\sum_{\pi \in N C(2 m-1)} \kappa_{\pi}\left(\sum_{i=1}^{n} X_{i}, \ldots, \sum_{i=1}^{n} X_{i}\right) \\
& =\sum_{\pi \in N C(2 m-1)} \sum_{i_{1}, \ldots, i_{2 m-1} \in[n]} \kappa_{\pi}\left(X_{i_{1}}, \ldots, X_{i_{2 m-1}}\right)=0 .
\end{aligned}
$$

Also note that

$$
\varphi\left(X_{i_{1}} \cdots X_{i_{2 m-1}}\right)=\sum_{\pi \in N C(2 m-1)} \kappa_{\pi}\left(X_{i_{1}}, \ldots, X_{i_{2 m-1}}\right)=0 .
$$

Inserting these identities into (3.3), with $r=2 m-1$ we obtain

$$
\begin{aligned}
0 & =\varphi\left(\left[\sum_{i=1}^{n} X_{i}\right]^{2 m-1}\left(X_{1}^{2}-X_{1} X_{2}-X_{2} X_{1}+X_{2}^{2}\right)\right) \\
& =2 \varphi\left(\left[\sum_{i=1}^{n} X_{i}\right]^{2 m-1} X_{1}^{2}\right)-2 \varphi\left(\left[\sum_{i=1}^{n} X_{i}\right]^{2 m-1} X_{1} X_{2}\right) .
\end{aligned}
$$

Let us observe that

$$
\begin{aligned}
\varphi\left(\left[\sum_{i=1}^{n} X_{i}\right]^{2 m-1} X_{1}^{2}\right)= & \varphi\left(X_{1}^{2 m+1}\right)+\sum_{\substack{i \in[n] \\
i \neq 1}} \varphi\left(X_{i}^{2 m-1} X_{1}^{2}\right) \\
& +\sum_{\operatorname{ker} \underline{i}<\hat{1}_{2 m-1}} \varphi\left(X_{i_{1}} \cdots X_{i_{2 m-1}} X_{1}^{2}\right) .
\end{aligned}
$$

Note that for $i \neq 1$, using freeness and induction hypothesis,

$$
\varphi\left(X_{i}^{2 m-1} X_{1}^{2}\right)=\varphi\left(X_{1}^{2 m-1}\right) \varphi\left(X_{1}^{2}\right)=0 .
$$


Now consider the term $\varphi\left(X_{i_{1}} \cdots X_{i_{2 m-1}} X_{1}^{2}\right)$ in (3.5) for $\operatorname{ker} \underline{i}<\hat{1}_{2 m-1}$. Let us express this moment as a sum of products of free cumulants using (2.1). Then each summand always has an odd free cumulant of order strictly smaller than $2 m+1$ as a factor. If this is a mixed free cumulant, then it is zero by freeness. If it is a pure free cumulant, then it vanishes by using Lemma 2.1, because all odd moments of order smaller than $2 m+1$ are assumed to be zero. Therefore, this moment is zero. Hence (3.5) reduces to

$$
\varphi\left(\left[\sum_{i=1}^{n} X_{i}\right]^{2 m-1} X_{1}^{2}\right)=\varphi\left(X_{1}^{2 m+1}\right) .
$$

By similar arguments, one can demonstrate that

$$
\varphi\left(\left[\sum_{i=1}^{n} X_{i}\right]^{2 m-1} X_{1} X_{2}\right)=0 .
$$

Using (3.5)-(3.8) in (3.4) we conclude $\varphi\left(X_{1}^{2 m+1}\right)=0$.

Now we consider the even moments. Suppose that

$$
\varphi\left(X_{1}^{2 r}\right)=C_{r}, \text { for } 1 \leq r \leq m
$$

We wish to show that

$$
\varphi\left(X_{1}^{2 m+2}\right)=C_{m+1} .
$$

Equation (3.3), with $r=2 m$ yields

$$
\varphi\left(\left[\sum_{i=1}^{n} X_{i}\right]^{2 m}\left(X_{1}-X_{2}\right)^{2}\right)=2 \varphi\left(\left[\sum_{i=1}^{n} X_{i}\right]^{2 m}\right) .
$$

Note that by induction hypothesis, the $2 r$-th moment equals $C_{r}$ for $r \leq m$. Thus the right side of (3.9) is

$$
\begin{aligned}
2 \varphi\left(\left[\sum_{i=1}^{n} X_{i}\right]^{2 m}\right) & =2 n \varphi\left(X_{1}^{2 m}\right)+2 \sum_{\operatorname{ker} \underline{i}<\hat{1}_{2 m}} \varphi\left(X_{i_{1}} \cdots X_{i_{2 m}}\right) \\
& =2 n C_{m}+2 \sum_{\text {ker } \underline{i}<\hat{1}_{2 m}} \varphi\left(X_{i_{1}} \cdots X_{i_{2 m}}\right) .
\end{aligned}
$$

The left-hand side of (3.9) can be expanded as

$$
\begin{array}{r}
2 \varphi\left(X_{1}^{2 m+2}\right)+2 \sum_{i \neq 1} \varphi\left(X_{i}^{2 m} X_{1}^{2}\right)+2 \sum_{\text {ker } \underline{i}<\hat{1}_{2 m}} \varphi\left(X_{i_{1}} \cdots X_{i_{2 m}} X_{1}^{2}\right) \\
-2 \sum_{\text {ker } \underline{i}<\hat{1}_{2 m}} \varphi\left(X_{i_{1}} \cdots X_{i_{2 m}} X_{1} X_{2}\right) .
\end{array}
$$

Observe, by freeness of $X_{1}$ and $X_{i}, i \neq 1, \varphi\left(X_{i}^{2 m} X_{1}^{2}\right)=\varphi\left(X_{i}^{2 m}\right) \varphi\left(X_{1}^{2}\right)=C_{m}$. So, we get that

$$
\begin{aligned}
& \varphi\left(X_{1}^{2 m+2}\right) \\
& =C_{m}+\sum_{\text {ker } \underline{i}<\hat{1}_{2 m}}\left[\varphi\left(X_{i_{1}} \cdots X_{i_{2 m}}\right)-\varphi\left(X_{i_{1}} \cdots X_{i_{2 m}} X_{1}^{2}\right)+\varphi\left(X_{i_{1}} \cdots X_{i_{2 m}} X_{1} X_{2}\right)\right] .
\end{aligned}
$$


Thus in order to prove $\varphi\left(X_{1}^{2 m+2}\right)=C_{m+1}$, we have to show that

$$
\begin{aligned}
& C_{m+1}-C_{m}= \\
& \sum_{\text {ker } \underline{i}<\hat{1}_{2 m}}\left[\varphi\left(X_{i_{1}} \cdots X_{i_{2 m}}\right)-\varphi\left(X_{i_{1}} \cdots X_{i_{2 m}} X_{1}^{2}\right)+\varphi\left(X_{i_{1}} \cdots X_{i_{2 m}} X_{1} X_{2}\right)\right] .
\end{aligned}
$$

Note that by induction hypothesis, the $2 r$-th moment equals $C_{r}$ for $r \leq m$. We also know from the earlier discussion that all odd free cumulants (mixed or pure) are zero. As a consequence, in view of the one-to-one correspondence between moments and free cumulants given in (2.1)-(2.3), we conclude that $\kappa_{2}\left(X_{1}\right)=1$ and all higher free cumulants as well as the mixed free cumulants up to order $2 m$ vanish.

Now we compute the right hand side of equation (3.10) in terms of free cumulants via equation (2.1).

$$
\begin{aligned}
\sum_{\operatorname{ker} \underline{i}<\hat{1}_{2 m}} & {\left[\varphi\left(X_{i_{1}} \cdots X_{i_{2 m}}\right)-\varphi\left(X_{i_{1}} \cdots X_{i_{2 m}} X_{1}^{2}\right)+\varphi\left(X_{i_{1}} \cdots X_{i_{2 m}} X_{1} X_{2}\right)\right] } \\
= & \sum_{\operatorname{ker} \underline{i}<\hat{1}_{2 m}}[\underbrace{\sum_{\pi \in N C_{2}(2 m)} \kappa_{\pi}\left(X_{i_{1}}, \ldots, X_{i_{2 m}}\right)}_{(\mathrm{I})} \\
& -\underbrace{\sum_{\pi \in N C_{2}(2 m+2)} \kappa_{\pi}\left(X_{i_{1}}, \ldots, X_{i_{2 m}}, X_{1}, X_{1}\right)}_{(\mathrm{II})} \\
& +\underbrace{\sum_{\pi \in N C_{2}(2 m+2)}}_{\sum_{\text {III })} \kappa_{\pi}\left(X_{i_{1}}, \ldots, X_{i_{2 m}}, X_{1}, X_{2}\right)} .
\end{aligned}
$$

We split (II) from (3.11) into two parts:

$$
\begin{aligned}
\sum_{\pi \in N C_{2}(2 m+2)} \kappa_{\pi}\left(X_{i_{1}}, \ldots, X_{i_{2 m}}, X_{1}, X_{1}\right)= & \sum_{\pi \in N_{1}} \kappa_{\pi}\left(X_{i_{1}}, \ldots, X_{i_{2 m}}, X_{1}, X_{1}\right) \\
& +\sum_{\pi \in N_{2}} \kappa_{\pi}\left(X_{i_{1}}, \ldots, X_{i_{2 m}}, X_{1}, X_{1}\right)
\end{aligned}
$$

where

$$
\begin{aligned}
& N_{1}=\left\{\pi \in N C_{2}(2 m+2) \mid 2 m+1 \stackrel{\pi}{\sim} 2 m+2\right\}, \\
& N_{2}=\left\{\pi \in N C_{2}(2 m+2) \mid 2 m+1 \stackrel{\pi}{\sim} 2 m+2\right\} .
\end{aligned}
$$

Recall that the contribution of each partition is either 0 or 1 , and $\kappa_{2}\left(X_{1}\right)=1$. Since by Fact $1, N_{1}$ is in bijection with $N C_{2}(2 m)$, we conclude that the contributions of $N_{1}$ and part (I) cancel out and we can rewrite (3.11) as

$\sum_{\operatorname{ker} \underline{i}<\hat{1}_{2 m}}\left[\sum_{\pi \in N C_{2}(2 m+2)} \kappa_{\pi}\left(X_{i_{1}}, \ldots, X_{i_{2 m}}, X_{1}, X_{2}\right)-\sum_{\pi \in N_{2}} \kappa_{\pi}\left(X_{i_{1}}, \ldots, X_{i_{2 m}}, X_{1}, X_{1}\right)\right]$.

We want to show that this equals $C_{m+1}-C_{m}$. First note that

$$
\sum_{\pi \in N C_{2}(2 m+2)} \kappa_{\pi}\left(X_{i_{1}}, \ldots, X_{i_{2 m}}, X_{1}, X_{2}\right)=\sum_{\pi \in N_{2}} \kappa_{\pi}\left(X_{i_{1}}, \ldots, X_{i_{2 m}}, X_{1}, X_{2}\right),
$$


since any $\pi \in N_{1}$ will contribute 0 due to free independence of $X_{1}$ and $X_{2}$. Interchanging the sums, we have to show

$$
\begin{gathered}
\sum_{\pi \in N_{2}} \sum_{\text {ker } \underline{i} \hat{1}_{2 m}}\left[\kappa_{\pi}\left(X_{i_{1}}, \ldots, X_{i_{2 m}}, X_{1}, X_{2}\right)-\kappa_{\pi}\left(X_{i_{1}}, \ldots, X_{i_{2 m}}, X_{1}, X_{1}\right)\right] \\
=C_{m+1}-C_{m} .
\end{gathered}
$$

Now note that

$$
\left|N_{2}\right|=C_{m+1}-C_{m}
$$

This is because $N_{1} \cap N_{2}=\emptyset$ and $N_{1} \cup N_{2}=N C_{2}(2 m+2)$. By Fact $1, \mid N C_{2}(2 m+$ $2) \mid=C_{m+1}$ and $\left|N_{1}\right|=\left|N C_{2}(2 m)\right|=C_{m}$. So, it is enough to show that the inner sum in (3.12) equals 1 for every $\pi \in N_{2}$.

Now write $\left\{\underline{i} \mid \operatorname{ker} \underline{i}<\hat{1}_{2 m}\right\}=B_{1} \cup B_{2}$, where $B_{1}$ contains only those multiindices whose components are either 1 or 2 , and $B_{2}$ contains the remaining multiindices. Fix $\pi \in N_{2}$. The inner sum on the left-hand side of (3.12) can therefore be written as a sum over indices in $B_{1}$ and a sum over indices in $B_{2}$. Both $\kappa_{\pi}\left(X_{i_{1}}, \ldots, X_{i_{2 m}}, X_{1}, X_{2}\right)$ and $\kappa_{\pi}\left(X_{i_{1}}, \ldots, X_{i_{2 m}}, X_{1}, X_{1}\right)$ cannot contribute for the same multi-index. This is because, in the first one, the $(2 m+2)$-th element $X_{2}$ should be paired with another $X_{2}$ in $\pi$, while in the second, the $(2 m+2)$-th argument $X_{1}$ should be paired with $X_{1}$, but both cannot happen simultaneously. Henceforth, for fixed $\pi$ we call a multi-index ker $\underline{i}<\hat{1}_{2 m}$ contributing if

$$
\kappa_{\pi}\left(X_{i_{1}}, \ldots, X_{i_{2 m}}, X_{1}, X_{2}\right)=1 \text { or } \kappa_{\pi}\left(X_{i_{1}}, \ldots, X_{i_{2 m}}, X_{1}, X_{1}\right)=1 .
$$

For $\pi \in N_{2}$, let

$$
I_{\pi, 1}:=\left\{\underline{i} \in \hat{1}_{2 m} \mid \operatorname{ker} i \circ(1,1) \geq \pi\right\} \text { and } I_{\pi, 2}:=\left\{\underline{i} \in \hat{1}_{2 m} \mid \operatorname{ker} i \circ(1,2) \geq \pi\right\} .
$$

Let $j$ be the index matched to $2 m+2$, i.e $(j, 2 m+2) \in \pi$. Let us look at $B_{2}$ first. Let $\underline{i} \in B_{2}$ be a contributing multi-index. If $\pi$ pairs the $(2 m+2)$-th component of $I_{\pi, 1}$ and $I_{\pi, 2}$ with $i_{j}=1$ (respectively 2 ), then

$\kappa_{\pi}\left(X_{i_{1}}, \ldots, X_{i_{2 m}}, X_{1}, X_{1}\right)=1$ (resp. 0) and $\kappa_{\pi}\left(X_{i_{1}}, \ldots, X_{i_{2 m}}, X_{1}, X_{2}\right)=0$ (resp. 1).

But then we also get another (unique) contributing multi-index, say, $\underline{k} \in B_{2}$ whose $j$-th component is 2 (respectively 1 ), while all other components are same as those in $I_{\pi, 1}$ or $I_{\pi, 2}$. For this $\underline{k}$ we have

$\kappa_{\pi}\left(X_{k_{1}}, \ldots, X_{k_{2 m}}, X_{1}, X_{1}\right)=0\left(\right.$ resp. 1) and $\kappa_{\pi}\left(X_{k_{1}}, \ldots, X_{k_{2 m}}, X_{1}, X_{2}\right)=1$ (resp. 0).

So for each fixed $\pi \in N_{2}$,

$$
\sum_{\underline{i} \in B_{2}}\left[\kappa_{\pi}\left(X_{i_{1}}, \ldots, X_{i_{2 m}}, X_{1}, X_{2}\right)-\kappa_{\pi}\left(X_{i_{1}}, \ldots, X_{i_{2 m}}, X_{1}, X_{1}\right)\right]=0 .
$$

Next we look at $B_{1}$. For the contributing $\underline{i} \in B_{1}$, with only one component 2 ,

$$
\kappa_{\pi}\left(X_{i_{1}}, \ldots, X_{i_{2 m}}, X_{1}, X_{2}\right)=1 \text { and } \kappa_{\pi}\left(X_{i_{1}}, \ldots, X_{i_{2 m}}, X_{1}, X_{1}\right)=0 .
$$

Observe that this multi-index has no corresponding counter-term in $B_{1}$, as to get the counter-term we need to change the 2 to 1 , but then all indices in the multiindex become 1 , contradicting the fact that $B_{1}$ is a subset of $\left\{\underline{i} \mid \operatorname{ker} \underline{i}<\hat{1}_{2 m}\right\}$. So for this particular multi-index, the contribution happens positively to (3.12). Any other contributing multi-index $\underline{i} \in B_{1}$ must have at least two 2's, and thus on 
inverting one of these 2 's to 1 , we still get a contributing multi-index in $B_{1}$, but with the opposite sign, leading to a cancellation. Thus for fixed $\pi \in N_{2}$,

$$
\sum_{\underline{i} \in B_{1}}\left[\kappa_{\pi}\left(X_{i_{1}}, \ldots, X_{i_{2 m}}, X_{1}, X_{2}\right)-\kappa_{\pi}\left(X_{i_{1}}, \ldots, X_{i_{2 m}}, X_{1}, X_{1}\right)\right]=1 .
$$

Therefore, for fixed $\pi \in N_{2}$,

$$
\sum_{\operatorname{ker} \underline{\underline{i}<\hat{1}_{2 m}}}\left[\kappa_{\pi}\left(X_{i_{1}}, \ldots, X_{i_{2 m}}, X_{1}, X_{2}\right)-\kappa_{\pi}\left(X_{i_{1}}, \ldots, X_{i_{2 m}}, X_{1}, X_{1}\right)\right]=1 .
$$

This completes the proof.

3.3. Zero correlation and free independence. In this section we first give a counterexample which shows that the free probability analogue of Theorem 1.3 is false. However, a "quantum" analogue, if we admit matrix coefficients, is indeed valid.

3.3.1. A counterexample to the scalar free analogue. As mentioned earlier, in classical probability, if a random vector $[X, Y]$ has a bivariate normal distribution and $\operatorname{Cov}(X, Y)=0$, then $X$ and $Y$ are independent. We now give an example where the above is not true if we replace "Gaussian" by "semicircular", and "independence" by "freeness". We need some basic concepts from bi-freeness for systems of non-commutative random variables (see Voiculescu, 2014 for more details). Let $H_{\mathbb{R}}$ be a separable real Hilbert space and let $H$ be its complexification with inner product $\langle\cdot, \cdot\rangle$ that is linear in its first argument and anti-linear in its second argument. When considering elements in $H_{\mathbb{R}}$, it holds true that $\langle x, y\rangle=\langle y, x\rangle$. Let $\mathcal{F}_{\text {fin }}(H)$ be the (algebraic) full Fock space over $H$ :

$$
\mathcal{F}_{\text {fin }}(H):=\bigoplus_{n=0}^{\infty} H^{\otimes n}
$$

with the convention that $H^{\otimes 0}=\mathbb{C} \Omega$ is a one-dimensional normed space along a unit vector $\Omega$ (called the vacuum vector). Note that elements of $\mathcal{F}_{\text {fin }}(H)$ are finite linear combinations of the elements from $H^{\otimes n}, n \in \mathbb{N} \cup\{0\}$, and we do not take the completion. We equip $\mathcal{F}_{\text {fin }}(H)$ with the direct sum of the canonical inner products

$$
\left\langle x_{1} \otimes \cdots \otimes x_{m}, y_{1} \otimes \cdots \otimes y_{n}\right\rangle:=\delta_{m, n} \prod_{i=1}^{n}\left\langle x_{i}, y_{i}\right\rangle .
$$

For $f \in H$ the left (resp. right) creation and annihilation linear operators $l^{*}(f)$ and $l(f)$ (resp. $r^{*}(f)$ and $r(f)$ )are defined on elementary tensors by

$$
\begin{aligned}
& l^{*}(f)\left(f_{1} \otimes \cdots \otimes f_{n}\right):=f \otimes f_{1} \otimes \cdots \otimes f_{n}, \\
& l^{*}(f) \Omega=f, \\
& l(f)\left(f_{1} \otimes \cdots \otimes f_{n}\right):=\left\langle f, f_{1}\right\rangle f_{2} \otimes \cdots \otimes f_{n}, \\
& l(f) f_{1}:=\left\langle f, f_{1}\right\rangle \Omega, \\
& l(f) \Omega=0, \\
& r^{*}(f)\left(f_{1} \otimes \cdots \otimes f_{n}\right):=f_{1} \otimes \cdots \otimes f_{n} \otimes f, \\
& r^{*}(f) \Omega=f, \\
& r(f)\left(f_{1} \otimes \cdots \otimes f_{n}\right):=\left\langle f, f_{n}\right\rangle f_{1} \otimes \cdots \otimes f_{n-1}, \\
& r(f) f_{1}:=\left\langle f, f_{1}\right\rangle \Omega,
\end{aligned}
$$




$$
r(f) \Omega=0,
$$

and linearly extended to $\mathcal{F}_{\text {fin }}(H)$. Consider the algebra generated by the abovedefined operators on the left and on the right as a subalgebra of $\mathcal{B}\left(\mathcal{F}_{\text {fin }}(H)\right)$, the space of all bounded linear operators on $\mathcal{F}_{\text {fin }}(H)$. When equipped with the vacuum expectation state $\langle\cdot \Omega, \Omega\rangle$, that is, $\tau(x)=\langle x \Omega, \Omega\rangle$, it becomes a rich noncommutative probability space. For example, $L_{f}:=l^{*}(f)+l(f) \in \mathcal{B}\left(\mathcal{F}_{\text {fin }}(H)\right)$ is a model for the semicircle law (see Voiculescu et al., 1992).

We now present the counterexample. The bounded self-adjoint operator

$$
G_{f}:=l^{*}(f)+l(f)+r^{*}(f)+r(f) \quad \text { for } f \in H_{\mathbb{R}},
$$

plays a crucial role in that.

Let us take $f, g \in H_{\mathbb{R}}, f, g \neq 0$ and $\langle f, g\rangle=0$. We claim that for all $a, b \in \mathbb{R}$ $a G_{f}+b G_{g}$ is a semicircle variable. Indeed, $a G_{f}+b G_{g}=G_{a f+b g}$ and observe that the actions of the left and right creation/annihilation operators have the same effect on the vacuum vector $\Omega$. That is,

$$
G_{a f+b g}^{n} \Omega=L_{2(a f+b g)}^{n} \Omega \text { for all } n \in \mathbb{N} .
$$

But we know that $L_{a f+b g}$ has semicircle distribution with respect to the vacuum state. Thus

$$
\left\langle G_{a f+b g}^{k} \Omega, \Omega\right\rangle=\left\langle L_{2(a f+b g)}^{k} \Omega, \Omega\right\rangle= \begin{cases}4^{n}\left(a^{2}\|f\|^{2}+b^{2}\|g\|^{2}\right)^{n} C_{n} & \text { if } k=2 n, \\ 0 & \text { if } k \text { is odd },\end{cases}
$$

and hence $G_{a f+b g}$ follows the semicircle law. In particular, $\tau\left(G_{f}\right)=\left\langle G_{f} \Omega, \Omega\right\rangle=$ $0, \tau\left(G_{g}\right)=\left\langle G_{g} \Omega, \Omega\right\rangle=0$. Now we compute

$$
\kappa_{2}\left(G_{f}, G_{g}\right)=\tau\left(G_{f} G_{g}\right)=\left\langle G_{f} G_{g} \Omega, \Omega\right\rangle .
$$

Note that

$$
G_{g} \Omega=l^{*}(g) \Omega+l(g) \Omega+r^{*}(g) \Omega+r(g) \Omega=2 g .
$$

Thus

$$
G_{f} G_{g} \Omega=2 G_{f}(g)=2(f \otimes g+g \otimes f+2\langle f, g\rangle \Omega)=2(f \otimes g+g \otimes f),
$$

since we assumed $\langle f, g\rangle=0$. Thus

$$
\kappa_{2}\left(G_{f}, G_{g}\right)=\langle 2(f \otimes g+g \otimes f), \Omega)=0 .
$$

Finally, it suffices to show that $G_{f}$ and $G_{g}$ are not free. This is supported by direct calculation of the fourth mixed moments, i.e. $\tau\left(G_{f} G_{g} G_{f} G_{g}\right)=\left\langle G_{f} G_{g} G_{f} G_{g} \Omega, \Omega\right\rangle=$ $8\|f\|^{2}\|g\|^{2}$, but $\left\langle G_{f} \Omega, \Omega\right\rangle=\left\langle G_{g} \Omega, \Omega\right\rangle=0$, which contradicts the definition of free independence.

We outline this last computation. We have already observed that $G_{f} G_{g} \Omega=$ $2(f \otimes g+g \otimes f)$. Thus

$$
G_{g} G_{f} G_{g} \Omega=2\left(g \otimes f \otimes g+g \otimes g \otimes f+2\|g\|^{2} f+2 f \otimes g \otimes g\right) .
$$

Since we finally take inner product between $G_{f} G_{g} G_{f} G_{g} \Omega$ and $\Omega$, the only term that would contribute from $G_{g} G_{f} G_{g} \Omega$ is easily seen to be $4\|g\|^{2} f$ and so

$$
\left\langle G_{f} G_{g} G_{f} G_{g} \Omega, \Omega\right\rangle=4\|g\|^{2}\left\langle G_{f}(f), \Omega\right\rangle=4\|g\|^{2} \times 2\|f\|^{2}=8\|f\|^{2}\|g\|^{2} .
$$


3.3.2. Polynomial matrix identities. Let $M_{n}(\mathbb{C})$ be the algebra of $n \times n$ matrices over $\mathbb{C}$ and $M_{n}^{s a}(\mathbb{C}):=\left\{A \mid A=A^{*}, A \in M_{n}(\mathbb{C})\right\}$. We say that a subset $A \subset M_{n}(\mathbb{C})$ satisfies a polynomial identity if there exists a polynomial $f \neq 0$ in non-commuting variables $x_{1}, \ldots, x_{d}$ for some $d \in \mathbb{N}$, such that $f\left(x_{1}, \ldots, x_{d}\right)=0$ for all $x_{1}, \ldots, x_{d} \in$ $A$. For example, the ring of $2 \times 2$ matrices over $\mathbb{C}$ satisfies the Hall identity

$$
f\left(x_{1}, x_{2}, x_{3}\right)=\left(x_{1} x_{2}-x_{2} x_{1}\right)^{2} x_{3}-x_{3}\left(x_{1} x_{2}-x_{2} x_{1}\right)^{2}=0 .
$$

In the following lemma we show that there is no universal polynomial identity which holds for all self-adjoint matrices. This extends a well-known result of Herstein (1968, Lemma 6.2.1) (the sets $M_{n}^{s a}(\mathbb{C}), n \in \mathbb{N}$ do not form an algebra, therefore we cannot directly apply this result). However the idea of our proof is germane in the arguments of Herstein (1968, Lemma 6.2.4). We provide the details for completeness.

Lemma 3.3. Suppose $d$ is a positive integer and $f \neq 0$ is a polynomial in noncommuting variables $x_{1}, \ldots, x_{d}$. Then there exists an $n$ such that $f$ is not a polynomial identity of $M_{n}^{s a}(\mathbb{C})$.

Proof: For a proof by contradiction, suppose there exists a polynomial $f \neq 0$ such that

$$
f\left(x_{1}, \ldots, x_{d}\right)=0 \text { for all } x_{1}, \ldots, x_{d} \in M_{n}^{s a}(\mathbb{C}) .
$$

By definition, a multilinear polynomial is a polynomial that is linear in each of its variables. In other words, no variable occurs to a power of 2 or higher; or alternatively, each monomial is a constant times a product of distinct variables. Now we observe that $M_{n}^{s a}(\mathbb{C})$ satisfies a multilinear identity of degree smaller than or equal to $d$. Indeed, $M_{n}^{s a}(\mathbb{C})$ also satisfies the identity

$$
\begin{aligned}
g\left(x_{1}, \ldots, x_{d}, x_{d+1}\right)= & f\left(x_{1}+x_{d+1}, x_{2}, \ldots, x_{d}\right) \\
& -f\left(x_{1}, x_{2}, \ldots, x_{d}\right)-f\left(x_{d+1}, x_{2} \ldots, x_{d}\right)=0 .
\end{aligned}
$$

Note that $g$ has a lower degree in $x_{1}$ and $x_{d+1}$ compared to $f$. Proceeding in this way, we can reduce our identity where only the first degree of $x_{1}$ is present. Now we go on to $x_{2}$ and repeat the procedure above. As we proceed through all the variables, we end up with a multilinear identity $\omega$. It is worth mentioning that the degree of the new polynomial is at most as large as that of $f$.

Now for any arbitrary $c_{1}, \ldots, c_{m} \in M_{n}(\mathbb{C})$, and their decomposition $c_{j}=a_{j}+i b_{j}$, $a_{j}, b_{j} \in M_{n}^{s a}(\mathbb{C})$, we have

$$
\omega\left(c_{1}, \ldots, c_{m}\right)=\sum_{d_{j_{1}} \in\left\{a_{j_{1}}, b_{j_{1}}\right\}, \ldots, d_{j_{m}} \in\left\{a_{j_{m}}, b_{j_{m}}\right\}} i^{\#\left\{p: d_{j_{p}}=b_{j_{p}}\right\}} \omega\left(d_{j_{1}}, \ldots, d_{j_{m}}\right)=0 .
$$

Thus we have constructed a non-zero polynomial identity which holds for all $M_{n}(\mathbb{C})$. But this contradicts the result given in Herstein (1968, Lemma 2.6.1) that such an identity cannot exist.

3.3.3. Generalization to the case with amalgamation. Freeness with amalgamation was introduced by Voiculescu (1995) as an extension of non-commutative probability spaces where matrices over a non-commutative probability space are considered.

Let $(\mathcal{A}, \varphi)$ be a non-commutative probability space as introduced in Section 2, and let $n$ be a positive integer. The algebra $M_{n}(\mathcal{A})$ of $n \times n$ matrices over $\mathcal{A}$ is a 
non-commutative probability space with canonical expectation

$$
\begin{aligned}
E^{\mathcal{B}}:=i d \otimes \varphi: M_{n}(\mathcal{A}) & \rightarrow M_{n}(\mathbb{C}) \\
A & \mapsto\left[\varphi\left(a_{i, j}\right)\right]_{i, j=1}^{n},
\end{aligned}
$$

where $A=\left[a_{i, j}\right]_{i, j=1}^{n}$ is a matrix in $M_{n}(\mathcal{A})$. Then $\left(M_{n}(\mathcal{A}), E^{\mathcal{B}}\right)$ is itself a noncommutative probability space. Observe that if we take $n=1$, then we are back to the scalar valued framework, discussed in Section 2.

We refer to Mingo and Speicher (2017, Ch. 9) for details. For $A_{1}, A_{2}, \ldots, A_{r} \in$ $M_{n}(\mathcal{A})$, its joint distribution is given by all joint moments of the form

$$
E^{\mathcal{B}}\left(A_{1}^{\prime} B_{1} A_{2}^{\prime} \cdots B_{k-1} A_{k}^{\prime}\right)
$$

where $A_{i}^{\prime} \in\left\{A_{1}, A_{2}, \ldots, A_{d}\right\}, B_{i} \in M_{n}(\mathbb{C})$ and $k \in \mathbb{N}$.

We define the operator-valued free cumulants $\kappa_{r}^{\mathcal{B}}: M_{n}(\mathcal{A})^{r} \rightarrow M_{n}(\mathbb{C})$ in the same way as in the scalar case:

$$
E^{\mathcal{B}}\left(A_{1} A_{2} \cdots A_{r}\right):=\sum_{\pi \in N C(r)} \kappa_{\pi}^{\mathcal{B}}\left(A_{1}, A_{2}, \ldots, A_{r}\right),
$$

but now cumulants are nested inside each other according to the nesting of blocks of $\pi$ (see Mingo and Speicher, 2017, Ch. 9 for more details).

A self-adjoint element $A \in M_{n}(\mathcal{A})$ is called an operator-valued semicircular element if its operator-valued moments $E^{\mathcal{B}}\left(A^{r}\right)$ have contributing terms in the above formula only through $\pi \in N C_{2}(r)$. This is equivalent to saying that $\kappa_{r}^{\mathcal{B}}(A)=0$ for $r \geq 3$.

Some special notations and facts. For $X \in \mathcal{A}$ we denote by $\left[\begin{array}{cc}X & 0 \\ 0 & X\end{array}\right]_{n}$ the element in $M_{n}(\mathcal{A})$ where the diagonal elements are equal to $X$ and the off-diagonal elements are equal to zero. The shift coefficient technique will be useful to us (this is in some sense the module property of the expectation and the cumulants; see Speicher, 1998). It implies that for $B_{i} \in M_{n}(\mathbb{C})$ and $A_{i} \in M_{n}(\mathcal{A})$, we have

$$
\begin{aligned}
& \kappa_{r}^{\mathcal{B}}\left(B_{1} A_{1}, B_{2} A_{2}, \ldots, B_{r-1} A_{r-1}, B_{r} A_{r}\right) \\
& =B_{1} \kappa_{r}^{\mathcal{B}}\left(A_{1} B_{2}, A_{2}, \ldots, A_{r-2} B_{r-1}, A_{r-1} B_{r}, A_{r}\right),
\end{aligned}
$$

and by induction if $B_{i}$ 's commute with all $A_{i}$ 's, then this equals

$$
B_{1} B_{2} \ldots B_{r} \kappa_{r}^{\mathcal{B}}\left(A_{1}, A_{2}, \ldots, A_{r}\right) .
$$

Moreover, let us note that

$$
\kappa_{r}^{\mathcal{B}}\left(\left[\begin{array}{cc}
X_{1} & 0 \\
0 & X_{1}
\end{array}\right]_{n}, \ldots,\left[\begin{array}{cc}
X_{r} & 0 \\
0 & X_{r}
\end{array}\right]_{n}\right)=\left[\begin{array}{ll}
1 & 0 \\
0 & 1
\end{array}\right]_{n} \kappa_{r}\left(X_{1}, \ldots, X_{r}\right)
$$

The last fact follows from the observation that $E^{\mathcal{B}}\left(\left[\begin{array}{cc}X_{1} & 0 \\ 0 & X_{1}\end{array}\right]_{n} \ldots\left[\begin{array}{cc}X_{r} & 0 \\ 0 & X_{r}\end{array}\right]_{n}\right)=$ $\left[\begin{array}{ll}1 & 0 \\ 0 & 1\end{array}\right]_{n} \varphi\left(X_{1} \cdots X_{r}\right)$.

We conclude with a matrix valued free version of Problem 3, which can be formulated as below. We would like to emphasize that now we have more information available because $A, B$ run through all of $M_{n}^{s a}(\mathbb{C})$. 
Theorem 3.4. Assume that we have two random variables $X, Y \in \mathcal{A}$, with the property that

(a) $\operatorname{Cov}(X, Y)=0$;

(b) $A \otimes X+B \otimes Y$ is an operator-valued semicircular element, for any pair $A, B \in$ $M_{n}^{s a}(\mathbb{C})$.

Then $X$ and $Y$ are free independent.

Proof: First we note that it suffices to show that mixed free cumulants of $X$ and $Y$ vanish. Our assumptions imply that

$$
\kappa_{r}^{\mathcal{B}}(A \otimes X+B \otimes Y)=0,
$$

for all $r \geq 3$, and self-adjoint matrices $A, B \in M_{n}(\mathbb{C})$. First, observe that

$$
D \otimes Z=D\left[\begin{array}{ll}
Z & 0 \\
0 & Z
\end{array}\right]_{n}=\left[\begin{array}{ll}
Z & 0 \\
0 & Z
\end{array}\right]_{n} D, \text { for } Z \in \mathcal{A} \text { and } D \in M_{n}(\mathbb{C}) \text {. }
$$

Thus by expanding equation (3.18) and using (3.16), (3.17) we may write, for $r \geq 3$,

$$
\begin{aligned}
0= & \sum_{\substack{C_{i_{1}}, \ldots, C_{i_{r}} \in\{A, B\} \\
Z_{i_{1}}, \ldots, Z_{i_{r}} \in\{X, Y\}}} C_{i_{1}} \ldots C_{i_{r}} \kappa_{r}^{\mathcal{B}}\left(\left[\begin{array}{cc}
Z_{i_{1}} & 0 \\
0 & Z_{i_{1}}
\end{array}\right], \ldots,\left[\begin{array}{cc}
Z_{i_{r}} & 0 \\
0 & Z_{i_{r}}
\end{array}\right]\right) \\
= & \sum_{\substack{C_{i_{1}}, \ldots, C_{i_{r}} \in\{A, B\} \\
Z_{i_{1}}, \ldots, Z_{i_{r}} \in\{X, Y\}}} C_{i_{1}} \ldots C_{i_{r}} \kappa_{r}\left(Z_{i_{1}}, \ldots, Z_{i_{r}}\right) .
\end{aligned}
$$

Now if we fix $r \geq 3$, then equation (3.19) holds for all $A, B \in M_{n}^{s a}(\mathbb{C})$, which by Lemma 3.3 implies that $\kappa_{r}\left(Z_{i_{1}}, \ldots, Z_{i_{r}}\right)=0$. Therefore, we conclude that all mixed free cumulants of at least third degree disappear. Now taking into account that $\kappa_{2}(X, Y)=\operatorname{Cov}(X, Y)=0$, we conclude that $X$ and $Y$ are free independent.

\section{Acknowledgements}

We are grateful to the Referee for encouraging us with her/his detailed and insightful comments. This has led to a significant improvement in our presentation. We thank Marek Bożejko and Franz Lehner for their valuable comments.

\section{References}

O. Arizmendi and A. Jaramillo. Convergence of the fourth moment and infinite divisibility: quantitative estimates. Electron. Commun. Probab. 19, no. 26, 12 (2014). MR3208324.

D. Basu. On statistics independent of a complete sufficient statistic. Sankhyā 15, 377-380 (1955). MR0074745.

M. Bożejko and W. Bryc. On a class of free Lévy laws related to a regression problem. J. Funct. Anal. 236 (1), 59-77 (2006). MR2227129.

W. Ejsmont. New characterization of two-state normal distribution. Infin. Dimens. Anal. Quantum Probab. Relat. Top. 17 (3), 1450019, 21 (2014). MR3241028.

W. Ejsmont, U. Franz and K. Szpojankowski. Convolution, subordination, and characterization problems in noncommutative probability. Indiana Univ. Math. J. 66 (1), 237-257 (2017). MR3623409.

W. Ejsmont and F. Lehner. Sample variance in free probability. J. Funct. Anal. 273 (7), 2488-2520 (2017). MR3677831. 
T. Hasebe. Free infinite divisibility for powers of random variables. ALEA Lat. Am. J. Probab. Math. Stat. 13 (1), 309-336 (2016). MR3481440.

I. N. Herstein. Noncommutative rings. The Carus Mathematical Monographs, No. 15. Published by The Mathematical Association of America; distributed by John Wiley \& Sons, Inc., New York (1968). MR0227205.

J. A. Mingo and R. Speicher. Free probability and random matrices, volume 35 of Fields Institute Monographs. Springer, New York; Fields Institute for Research in Mathematical Sciences, Toronto, ON (2017). ISBN 978-1-4939-6941-8; 978-14939-6942-5. MR3585560.

A. Nica and R. Speicher. Lectures on the combinatorics of free probability, volume 335 of London Mathematical Society Lecture Note Series. Cambridge University Press, Cambridge (2006). ISBN 978-0-521-85852-6; 0-521-85852-6. MR2266879.

J. C. Pardo, J.-L. Pérez and V. Pérez-Abreu. On the non-commutative fractional Wishart process. J. Funct. Anal. 272 (1), 339-362 (2017). MR3567507.

E. Seneta and G. J. Szekely. Normal characterization by zero correlations. J. Aust. Math. Soc. 81 (3), 351-361 (2006). MR2300161.

R. Speicher. Combinatorial theory of the free product with amalgamation and operator-valued free probability theory. Mem. Amer. Math. Soc. 132 (627), x+88 (1998). MR1407898.

K. Szpojankowski. On the Lukacs property for free random variables. Studia Math. 228 (1), 55-72 (2015). MR3416954.

K. Szpojankowski. On the Matsumoto-Yor property in free probability. J. Math. Anal. Appl. 445 (1), 374-393 (2017). MR3543772.

K. Szpojankowski and J. Wesołowski. Dual Lukacs regressions for non-commutative variables. J. Funct. Anal. 266 (1), 36-54 (2014). MR3121719.

D. Voiculescu. Addition of certain noncommuting random variables. J. Funct. Anal. 66 (3), 323-346 (1986). MR839105.

D. Voiculescu. Limit laws for random matrices and free products. Invent. Math. 104 (1), 201-220 (1991). MR1094052.

D. Voiculescu. Operations on certain non-commutative operator-valued random variables. Astérisque (232), 243-275 (1995). Recent advances in operator algebras (Orléans, 1992).

D. Voiculescu. Free probability for pairs of faces I. Comm. Math. Phys. 332 (3), 955-980 (2014). MR3262618.

D. V. Voiculescu, K. J. Dykema and A. Nica. Free random variables, volume 1 of CRM Monograph Series. American Mathematical Society, Providence, RI (1992). ISBN 0-8218-6999-X. MR1217253. 